\title{
Maximal sets of integers not containing $k+1$ pairwise coprimes and having divisors from a specified set of primes
}

\author{
Vladimir Blinovsky \\ ${ }^{1}$ Bielefeld University, Math. Dept., P.O.100131,D-33501, Bielefeld, Germany,vblinovs@math.uni-bielefeld.de
}

\begin{abstract}
We find the formula for the cardinality of maximal set of integers from $[1, \ldots, n]$ which does not contain $k+1$ pairwise coprimes and has divisors from a specified set of primes. This formula is defined by the set of multiples of the generating set, which does not depend on $n$.
\end{abstract}

Keywords: greatest common divisor, coprimes, squarefree numbers

\section{Formulation of the result}

Let $\mathbb{P}=\left\{p_{1}<p_{2}, \ldots\right\}$ be the set of primes and $\mathbb{N}$ be the set of natural numbers. Write $\mathbb{N}(n)=$ $\{1, \ldots, n\}, \mathbb{P}(n)=\mathbb{P} \bigcap \mathbb{N}(n)$. For $a, b \in \mathbb{N}$ denote the greatest common divisor of $a$ and $b$ by $(a, b)$. Let $S(n, k)$ be the family of sets $A \subset \mathbb{N}(n)$ of positive integers which does not contain $k+1$ coprimes. Define

$$
f(n, k)=\max _{A \in S(n, k)}|A| .
$$

In the paper [1] the following was proved.

Theorem 1 For all sufficiently large

$$
f(n, k)=|\mathbb{E}(n, k)|
$$

where

$$
\mathbb{E}(n, k)=\left\{a \in \mathbb{N}(n): a=u p_{i}, \text { for some } i=1, \ldots, k\right\} .
$$

Let now $\mathbb{Q}=\left\{q_{1}<q_{2}<\ldots<q_{r}\right\} \subset \mathbb{P}$ be finite set of primes and $R(n, \mathbb{Q}) \subset S(n, 1)$ is such family of sets of positive integers that for the arbitrary $a \in A \in R(n, \mathbb{Q}),\left(a, \prod_{j=1}^{r} q_{j}\right)>1$. In [2] was proved the following

Theorem 2 Let $n \geq \prod_{j=1}^{r} q_{j}$, then

$$
f(n, \mathbb{Q}) \triangleq \max _{A \in R(n, \mathbb{Q})}|A|=\max _{1 \leq t \leq r}\left|M\left(2 q_{1}, \ldots, 2 q_{t}, q_{1} \ldots q_{t}\right) \bigcap \mathbb{N}(n)\right|,
$$

where $M(B)$ is the set of multiples of the set of integers $B$.

1365-8050 (c) 2005 Discrete Mathematics and Theoretical Computer Science (DMTCS), Nancy, France 
In [2] the problem was stated of finding the maximal set of positive integers from $\mathbb{N}(n)$ which satisfies the conditions of Theorems 1 and 2 simultaneously i.e. which is a set $A$ without $k+1$ coprimes and such that each element of this set has a divisor from $\mathbb{Q}$. This paper is devoted to the solution of this problem. In our work we use the methods from the paper [1].

Denote $R(n, k, \mathbb{Q}) \subset S(n, k)$ the family of sets of positive integers with the property that an arbitrary $a \in A \in R(n, k, \mathbb{Q})$ has divisor from $\mathbb{Q}$. For given $s$ and $\mathbb{T}=\left\{r_{1}<r_{2}<\ldots\right\}=\mathbb{P}-\mathbb{Q}$ let $F(n, k, s, \mathbb{Q}) \subset R(n, k, \mathbb{Q})$ is the family of sets of squarefree positive numbers such that for the arbitrary $a \in A \in F(n, k, s, \mathbb{Q})$ we have $\left(r_{i}, a\right)=1, i>s$. For given $s, r$ cardinality of the family $F(n, k, s, \mathbb{Q})$ and cardinalities of each $A \in F(n, k, s, \mathbb{Q})$ are bounded from above as $n \rightarrow \infty$.

Next we formulate our main result which extent the result of the Theorems 1,2 and in some sense include both of them.

Theorem 3 If $\mathbb{Q} \neq \emptyset$, then for sufficiently large $n$ the following relation is valid

$$
\varphi(n, k, \mathbb{Q}) \triangleq \max _{A \in R(n, k, \mathbb{Q})}|A|=\max _{F \in F(n, k, s-1, \mathbb{Q})}|M(F) \bigcap \mathbb{N}(n)|,
$$

where $s$ is the minimal integer which satisfies the inequality $r_{s}>r$.

\section{Proof of the Theorem 3}

Let's remind the definition of the left pushing which the reader can find in [2]. For the arbitrary

$$
a=u p_{j}^{\alpha}, p_{i}<p_{j},\left(p_{i} p_{j}, u\right)=1, \alpha>0 \text { and } p_{j} \notin \mathbb{Q} \text { or } p_{i}, p_{j} \in \mathbb{Q}
$$

define

$$
L_{i, j}(a, \mathbb{Q})=p_{i}^{\alpha} u .
$$

For $a$ not of the form (4) we set $L_{i, j}(a, \mathbb{Q})=a$. For $A \subset \mathbb{N}$ denote

$$
L_{i, j}(a, A, \mathbb{Q})= \begin{cases}L_{i, j}(a, \mathbb{Q}), & L_{i, j}(a, \mathbb{Q}) \notin A, \\ a, & L_{i, j}(a, \mathbb{Q}) \in A .\end{cases}
$$

At last set

$$
L_{i, j}(A, \mathbb{Q})=\left\{L_{i, j}(a, A, \mathbb{Q}) ; a \in A\right\} .
$$

We say that $A$ is left compressed if for the arbitrary $i<j$

$$
L_{i, j}(A, \mathbb{Q})=A \text {. }
$$

It can be easily seen that every finite $A \subset \mathbb{N}$ after finite number of left pushing operations can be made left compressed,

$$
\left|L_{i, j}(A, \mathbb{Q})\right|>|A|
$$

and if $A \in R(n, k, \mathbb{Q})$, then $L_{i, j}(A, \mathbb{Q}) \in R(n, k, \mathbb{Q})$.

If we denote $O(n, k, \mathbb{Q}) \subset R(n, k, \mathbb{Q})$ the families of sets on which achieved max in (3) and $C(n, k, \mathbb{Q}) \subset$ $R(n, k, \mathbb{Q})$ is the family of left compressed sets from $R(n, k, \mathbb{Q})$, then it follows that $O(n, k, \mathbb{Q}) \cap C(n, k, \mathbb{Q}) \neq$ $\emptyset$. Next we assume that $A \in C(n, k, \mathbb{Q}) \bigcap O(n, k, \mathbb{Q})$. 
For the arbitrary $a \in A$ we have the decomposition $a=a^{1} a^{2}$, where $a^{1}=r_{i_{1}}^{\alpha_{1}} \ldots r_{i_{f}}^{\alpha_{f}}, r_{i}<r_{j}, i<$ $j, a^{2}=q_{j_{1}}^{\beta_{1}} \ldots q_{j_{\ell}}^{\beta_{\ell}} ; q_{j_{m}}<q_{j_{s}}, m<s ; \alpha_{j}, \beta_{j}>0$. If $a=r_{i_{1}}^{\alpha_{1}} \ldots r_{i_{f}}^{\alpha_{f}} q_{j_{1}}^{\beta_{1}} \ldots q_{j_{\ell}}^{\beta_{\ell}} \in A, \alpha_{j}, \beta_{j}>0$,then $\bar{a}=r_{i_{1}} \ldots r_{i_{f}} q_{j_{1}} \ldots q_{j_{\ell}} \in A$ as well and also $\hat{a}=u a \in A$ for all $u \in \mathbb{N}: u a \leq n$. Consider all squarefree numbers $A^{*} \subset A$ and for given $a^{2}$ the set of all $a^{1}$ such that $a^{1} a^{2} \in A^{*}$. This set is the ideal generated by the division. The set of minimal elements from this ideal denote by $P\left(a^{2}, A^{*}\right)$. It follows that $(A \in O(n, k, \mathbb{N}))$,

$$
A=M\left(\left\{a^{1} a^{2} ; a^{1} \in P\left(a^{2}, A^{*}\right)\right\}\right) \bigcap \mathbb{N}(n),
$$

For each $a^{2}$ we order $\left\{a_{1}^{1}<a_{2}^{1}<\ldots\right\}=P\left(a^{2}, A^{*}\right)$ lexicographically according to their decomposition $a_{i}^{1}=r_{i_{1}} \ldots r_{i_{f}}$. Let $\rho$ is the maximal over the choice of $a^{2}$ positive integer such that $r_{\rho}$ divide some $a_{i}^{1}$ for which $a_{i}^{1} a^{2} \in A^{*}$. From the left compressedness of the set $A$ it follows that $a^{\prime}=a_{j}^{1} a^{2}, j<i$ also belongs to $A$. Then the set $B$ of elements $b=b^{1} b^{2} \leq n,\left(b^{1}, \prod_{j=1}^{r} q_{j}\right)=1$ such that $b^{2}=q_{j_{1}}^{\beta_{1}} \ldots q_{j_{\ell}}^{\beta_{\ell}}, \beta_{j}>0$ and $a_{i}^{1} \mid b^{1}, a_{j}^{1} \backslash b^{1}, j<i$ is exactly the set

$$
B(a)=\left\{u \leq n: u=r_{i_{1}}^{\alpha_{1}} \ldots r_{i_{f}}^{\alpha_{f}} r_{\rho}^{\alpha_{\rho}} q_{j_{1}}^{\beta_{1}} \ldots q_{j_{\ell}}^{\beta_{\ell}} F ; \alpha_{i}, \beta_{i}>0, \quad\left(F, \prod_{j=1}^{\rho} r_{j} \prod_{j=1}^{r} q_{j}\right)=1\right\} .
$$

Denote

$$
\begin{gathered}
P^{\rho}\left(a^{2}, A^{*}\right)=\left\{a \in P\left(a^{2}, A^{*}\right):\left(a, r_{\rho}\right)=r_{\rho}\right\}, \\
P_{s}^{\rho}\left(A^{*}\right)=\left\{a \in P^{\rho}\left(a^{2}, A^{*}\right) \text { for some } a^{2}, \text { such that }\left(a^{2}, \prod_{j=1}^{s} q_{j}\right)=q_{s}\right\}
\end{gathered}
$$

and

$$
L^{\rho}\left(a^{2}\right)=\bigcup_{a \in P^{\rho}\left(a^{2}, A^{*}\right)} B(a) .
$$

Then the set $\bigcup_{s=1}^{r} P_{s}^{\rho}\left(A^{*}\right)$ is exactly the set $\bigcup_{a^{2}} P^{\rho}\left(a^{2}, A^{*}\right)$ of numbers which are divisible by $r_{\rho}$. Because each $a \in P\left(a^{2}, A^{*}\right)$ for all $a^{2}$ has divisor from $\mathbb{Q}$ it follows that for some $1 \leq s \leq r$

$$
\left|\bigcup_{a \in P_{s}^{\rho}\left(A^{*}\right)} B(a)\right| \geq \frac{1}{r}\left|\bigcup_{a^{2}} L^{\rho}\left(a^{2}\right)\right| .
$$

Next for this $s$ we define the transformation

$$
\bar{P}\left(a^{2}, A^{*}\right)=\left(P\left(a^{2}, A^{*}\right)-P^{\rho}\left(a^{2}, A^{*}\right)\right) \bigcup R_{s}^{\rho}\left(a^{2}, A^{*}\right),
$$

where

$$
\begin{aligned}
& R_{s}^{\rho}\left(a^{2}, A^{*}\right)=\left\{v \in \mathbb{N} ; v r_{\rho} \in P_{s}^{\rho}\left(a^{2}, A\right)\right\} \\
& P_{s}^{\rho}\left(a^{2}, A^{*}\right)=\left\{a=a^{1} a^{2} \in P_{s}^{\rho}\left(A^{*}\right)\right\} .
\end{aligned}
$$

It is easy to see that

$$
\bigcup_{a^{2}} \bar{P}\left(a^{2}, A^{*}\right) \subset S(n, k, \mathbb{Q}) .
$$


Next we prove that if $r_{\rho}>r$, then

$$
\left|M\left(\bigcup_{a^{2}} \bar{P}\left(a^{2}, A^{*}\right)\right) \bigcap \mathbb{N}(n)\right|>|A|
$$

which gives the contradiction to the maximality of $A$.

For $a \in R_{s}^{\rho}\left(a^{2}, A^{*}\right), a=r_{i_{1}} \ldots r_{i_{f}} q_{j_{1}} \ldots q_{j_{\ell}}, r_{i_{1}}<\ldots<r_{i_{f}}<r_{\rho}, q_{j_{1}} \ldots q_{j_{\ell}}=a^{2}$ denote

$$
D(a)=\left\{v \in \mathbb{N}(n): v=r_{i_{1}}^{\alpha_{1}} \ldots r_{i_{f}}^{\alpha_{f}} q_{j_{1}}^{\beta_{1}} \ldots q_{j_{\ell}}^{\beta_{\ell}} T, \alpha_{j}, \beta_{j} \geq 1,\left(T, \prod_{j=1}^{\rho-1} r_{j} \prod_{j=1}^{r} q_{j}\right)=1\right\} .
$$

It can be easily seen that

$$
D(a) \bigcap D\left(a^{\prime}\right)=\emptyset, a \neq a^{\prime}
$$

and

$$
M\left(\bigcup_{a^{2}}\left(P\left(a^{2}, A^{*}\right)-P^{\rho}\left(a^{2}, A^{*}\right)\right)\right) \bigcap D(a)=\emptyset .
$$

Thus to prove (6) it is sufficient to show, that for large $n>n_{0}$

$$
|D(a)>r| B\left(a r_{\rho}\right) \mid \text {. }
$$

To prove (7) we consider three cases.

First case when $n /\left(a r_{\rho}\right) \geq 2$ and $\rho>\rho_{0}$. It follows that

$$
\begin{aligned}
\left|B\left(a r_{\rho}\right)\right| & \leq c_{2} \sum_{\alpha_{i}, \alpha, \beta_{i} \geq 1} \frac{n}{r_{i_{1}}^{\alpha_{1}} \ldots r_{i_{f}}^{\alpha_{f}} r_{\rho}^{\alpha} q_{j_{1}}^{\beta_{1}} \ldots q_{j_{\ell}}^{\beta_{\ell}}} \prod_{j=1}^{\rho}\left(1-\frac{1}{r_{j}}\right) \prod_{j=1}^{r}\left(1-\frac{1}{q_{j}}\right) \\
& =c_{2} \frac{n}{\left(r_{i_{1}}-1\right) \ldots\left(r_{i_{f}}-1\right)\left(r_{\rho}-1\right)\left(q_{j_{1}}-1\right) \ldots\left(q_{j_{\ell}}-1\right)} \prod_{j=1}^{\rho}\left(1-\frac{1}{r_{j}}\right) \prod_{j=1}^{r}\left(1-\frac{1}{q_{j}}\right) .
\end{aligned}
$$

At the same time

$$
\bar{D}(a) \triangleq\left\{v \in \mathbb{N}(n) ; v=r_{i_{1}} \ldots r_{i_{f}} q_{j_{1}} \ldots q_{j_{\ell}} F_{1}, \quad\left(F_{1}, \prod_{j=1}^{\rho-1} r_{j} \prod_{j=1}^{r} q_{j}\right)=1\right\} \subset D(a)
$$

and we obtain the inequalities

$$
|D(a)| \geq|\bar{D}(a)| \geq c_{1} \frac{n}{r_{i_{1}} \ldots r_{i_{f}} q_{j_{1}} \ldots q_{j \ell}} \prod_{j=1}^{\rho-1}\left(1-\frac{1}{r_{j}}\right) \prod_{j=1}^{r}\left(1-\frac{1}{q_{j}}\right) .
$$

Thus from (8), (9) it follows that

$$
\begin{aligned}
\frac{|D(a)|}{B\left(a r_{\rho}\right)} & \geq \frac{c_{1}}{c_{2}} r_{\rho} \frac{\left(r_{i_{1}}-1\right) \ldots\left(r_{i_{f}}-1\right)}{r_{i_{1}} \ldots r_{i_{f}}} \prod_{j \in[r]-\left\{j_{1}, \ldots, j_{\ell}\right\}}\left(1-\frac{1}{q_{j}}\right) \\
& \geq \frac{c_{1}}{c_{2}} \prod_{j=1}^{f}\left(1-\frac{1}{r_{j}}\right) r_{\rho} \prod_{j=1}^{r}\left(1-\frac{1}{q_{j}}\right)>r .
\end{aligned}
$$


Now let's $n /\left(a r_{\rho}\right) \geq 2, \rho<\rho_{0}$. Then we obtain the inequalities

$$
\begin{aligned}
\left|B\left(a r_{\rho}\right)\right| & <(1+\epsilon) \frac{n}{\left(r_{i_{1}}-1\right) \ldots\left(r_{i_{f}}-1\right)\left(r_{\rho}-1\right)\left(q_{j_{1}}-1\right) \ldots\left(q_{j_{\ell}}-1\right)} \prod_{j=1}^{\rho}\left(1-\frac{1}{r_{j}}\right) \prod_{j=1}^{r}\left(1-\frac{1}{q_{j}}\right), \\
|D(a)| & >(1-\epsilon) \frac{n}{\left(r_{i_{1}}-1\right) \ldots\left(r_{i_{f}}-1\right)\left(q_{j_{1}}-1\right) \ldots\left(q_{j_{\ell}}-1\right)} \prod_{j=1}^{\rho-1}\left(1-\frac{1}{r_{j}}\right) \prod_{j=1}^{r}\left(1-\frac{1}{q_{j}}\right) .
\end{aligned}
$$

From these inequalities it follows that

$$
\frac{|D(a)|}{\left|B\left(a r_{\rho}\right)\right|}>\frac{1-\epsilon}{1+\epsilon} r_{\rho}>r
$$

Here the last inequality is valid for sufficiently small $\epsilon$ because $r_{\rho}>r$.

The last case is when $1 \leq n /\left(a r_{\rho}\right)<2$. In this case $\left|B\left(a r_{\rho}\right)\right|=1$. Let's $r_{i_{1}} \ldots r_{i_{f}} r_{\rho} q_{j_{1}} \ldots q_{j_{\ell}}=$ $B\left(a r_{\rho}\right)$. Then we choose $r_{g}>\left(q_{j_{1}}\right)^{r}$ and $n>\prod_{j=1}^{q} r_{j} \prod_{j=1}^{r} q_{j}$. We have $r_{\rho}>r_{g}$. Indeed, otherwise

$$
n>\prod_{j=1}^{g} r_{j} \prod_{j=1}^{r} q_{j}>2 \prod_{j=1}^{\rho} \prod_{j=1}^{r} q_{j}>2 a r_{\rho}
$$

which is the contradiction to our case.

Hence

$$
\begin{aligned}
& \left\{r_{i_{1}} \ldots r_{i_{f}} q_{j_{1}} \ldots q_{j_{\ell}}, r_{i_{1}} \ldots r_{i_{f}} q_{j_{1}}^{2} \ldots q_{j_{\ell}},\right. \\
& \left.\ldots, r_{i_{1}} \ldots r_{i_{f}} q_{j_{1}}^{r} \ldots q_{j_{\ell}}, r_{i_{1}} \ldots r_{i_{f}} q_{j_{1}} \ldots q_{j_{\ell}} r_{\rho}\right\} \subset D(a) .
\end{aligned}
$$

Thus in this case also $|D(a)|>r=r\left|B\left(a_{\rho}\right)\right|$.

From the above follows that for sufficiently large $n>n_{0}(\mathbb{Q})$ for all $a \in R_{s}^{\rho}\left(a^{2}, A^{*}\right)$ inequality (7) is valid and taking into account (5) we obtain (6). This gives the contradiction to the maximality of $A$. Hence the maximal $r_{\rho} \in \mathbb{P}-\mathbb{Q}$ which appear as the divisor of some $a \in \bigcup_{a^{2}} P\left(a^{2}, A^{*}\right)$ such that $M\left(A^{*}\right) \cap \mathbb{N}(n) \in O(n, k, \mathbb{Q})$ satisfies the condition $r_{\rho} \leq r$. This inequality gives the statement of Theorem.

This is joint work with R.Ahlswede.

\section{References}

[1] R.Ahlswede and L.Khachatrian, Maximal sets of numbers not containing $k+1$ pairwise coprime integers, Acta Arithm., LXXII.1, 1995, 77-100

[2] R.Ahlswede and L.Khachatrian, Sets of integers with pairwise common divisor and a factor from a specified set of primes, Acta Arithm., LXXV.3, 1996, 259-276

[3] H.Halberstam and K.Roth, Sequences, Oxford University Press, 1966

[4] T.Apostol, Introduction to Analytic Number Theory, Springer-Verlag, N.Y., Berlin, 1976 
